\title{
Mental health among COVID-19 survivors and healthcare workers exposed to COVID-19 in Wuhan, China: a cross-sectional study
}

\section{Bin Chen}

affiliated hospital of nanjing university of chinese medicine

\section{Yan Wang}

affiliated hospital of nanjing university of chinese medicine

\section{Ting Yang}

affiliated hospital of nanjing university of chinese medicine

Cheng Li

affiliated hospital of nanjing university of chinese medicine

Zhiling Sun ( $\sim 20171552 @ n j u c m . e d u . c n$ )

Nanjing University of Chinese Medicine https://orcid.org/0000-0002-0315-2347

\section{Research}

Keywords: Mental health, COVID-19, SCL90-R, Survivors, Healthcare workers

Posted Date: May 26th, 2020

DOl: https://doi.org/10.21203/rs.3.rs-30351/v1

License: (c) (1) This work is licensed under a Creative Commons Attribution 4.0 International License. Read Full License 


\section{Abstract}

Then novel coronavirus disease (COVID-19) epidemic was considered to be the worst and complex virus outbreak, which caused 56,985 deaths as of April 22, 2020 already. The epidemic infectious may cause mental health crisis. Meanwhile, little is known about the specific psychological status of the COVID-19 survivors and healthcare workers. This cross-sectional study surveyed the mental health among 20 COVID-19 survivors, 54 nurses, and 24 hygienists in Wuhan, China and analyze the possible impact factors using the Symptom Check List 90 - Revised (SCL90-R) questionnaire. 3 indices and 9 dimensions were compared among job, education level, gender, age, marriage classification. This study found that mental distress among participants was not very serious in general. The survivors presented a highest score, then the hygienists, and the lowest in nurses. Low-educated and women showed significant increase. No significant difference was noted in age and marriage classification. Our study indicated that the survivors need psychological support immediately. Meanwhile, healthcare workers warrant more attention, especially low-educated and women. Comprehensive emergency response plan was warranted.

\section{Introduction}

The novel coronavirus disease (COVID-19) was first reported in Wuhan, China in December, 2019 ${ }^{1}$. After a latent period of 1-14 days (mostly 3-7 days), most patients infected with COVID-19 had clinical manifestations of fever, dry cough, and fatigue ${ }^{2}$. Due to the person-to-person transmission via respiratory droplets when patients coughed or sneezed and close contact ${ }^{3}$, the COVID-19 outbreak rapidly across China $^{4}$. As to April 4, 2020, source told by the National Health Commission of China, the cumulative number of confirmed patients was up to 82,798 (50,333 in Wuhan), including 4,632 death (3,869 in Wuhan $)^{5}$. Meanwhile, the virus erupted in other countries which causing outbreak globally. The World Health Organization released the latest situation report 1,051,635 confirmed cases and 56,985 deaths on April 22, $2020^{6}$.

Sustained growth of infections and death caused social fear and panic ${ }^{7}$. During the treatment, isolation made patients gradually alienate to each other. On the other hand, uncertainty of disease prognosis and the threat of death, psychological health problems like depression or anxiety were more likely to occur and worsen ${ }^{8}$. A study found that COVID-19 confirmed patients suffered severe mental distress even after rehabilitation and discharge ${ }^{9}$. These mental health problems may lead to some bad results, such as reducing the quality of life and affecting work performance ${ }^{10}$. Previous studies showed that during the period of Severe Acute Respiratory Syndrome (SARS) outbreak in 2003, proportion of SARS survivors in mental distress status was $9.8 \%$ in the beginning, what was worse, it has increased to $25.6 \%$ after 30 months ${ }^{11}$. This reminded us paying attention to mental health of COVID-19 survivors, yet, recent studies mainly focused on medical treatment and virus prevention.

Furthermore, healthcare workers rushing to rescue Wuhan also faced great pressure, including high risk of infection, inadequate of personal protection equipment, no specific medication, heavy workload, isolation, 
negative emotions of patients, lack of contact with family members, and fatigue ${ }^{12}$. These acute conditions may lead to severe psychological health status such as stress, anxiety, depression, insomnia, and fear. These mental health problems will not only affect the attention, understanding and decisionmaking ability of healthcare workers, may hamper them from fighting against COVID-19, but also have a lasting influence on their overall health ${ }^{13}$. Therefore, protecting the mental health of frontline healthcare workers was of great significance to control the epidemic situation and their own long-term health.

In view of the severe mental health problems, the National Health Commission of China published guidelines for emergency psychological intervention for COVID-19 survivors and healthcare workers ${ }^{14}$. Specific methods included establishment of psychological intervention working groups, psychological assistance hotlines, online mental health services, etc ${ }^{4,10,15}$. However, due to the unsatisfactory overall planning, lack of communications among medical services and mental health institutions, shortage of professional and experienced psychologists and psychiatrists ${ }^{16}$, survivors and healthcare workers didn't acquire enough psychological support.

To solve the psychological health problems, we need to make clear of the mental health status of COVID19 survivors and healthcare workers, and the impacting factors. The Symptom Check List 90 - Revised (SCL90-R) questionnaire was a multidimensional self-reported symptom scale which was used to estimate participants' psychological status ${ }^{17}$. Indeed, it was widely used in clinical research of medicine since its naissance ${ }^{18}$. Therefore, we quoted SCL90-R questionnaire to measure mental health status of survivors and healthcare workers, which could provide valuable evidence for psychological intervention among COVID-19 survivors and healthcare workers.

\section{Materials And Methods}

\section{Study design and participants}

This study is a cross-sectional, survey-based study which collected demographic information and mental health data via SCL90-R questionnaire from March 15 to 25, 2020 in Wuhan, China. A total of 20 COVID19 survivors and 78 healthcare workers were enrolled in the study. All participants were willing to complete the questionnaire, without mental disorders, 18 years of age or older, and other inclusion criteria were able to understand, speak, and write Chinese in order to finish the SCL90-R questionnaire smoothly. The study was approved by the Medical Ethics Committee of Affiliated Hospital of Nanjing University of Chinese Medicine. Written informed consent was provided by all enrolled participants before the initial of study, and all subjects were informed that this study was anonymous, all information was confidential, and participants had the right to end the questionnaire anytime without responsibility.

We investigated 78 healthcare workers from Wuhan No.1 Hospital, the Central Hospital of Wuhan, Wuhan Jiangxia District Hospital of Traditional Chinese Medicine, Cabin Hospital of Jiangxia District which was transformed from a gymnasium, Tongji Hospital, and Wuhan Jinyintan Hospital respectively. All of them were rushed to the rescue of Wuhan responding to the call of the Chinese government from multiple 
hospitals of Jiangsu Province, China from February to March 2020, consisting of 54 nurses and 24 hygienists.

20 patients with conforming COVID-19 were also surveyed in our study, who were admitted and treated in Cabin Hospital of Jiangxia District in March 2020. The included patients were diagnosed as COVID-19 and were discharged from the Cabin Hospital after recovery. Patients combined other diseases such as enteritis, or pulmonary occupying lesions were ruled out. The diagnostic criteria of COVID-19 were suspected case also has one of the following etiological or serological evidence: 1 . The COVID-19 nucleic acid was identified positively by real-time fluorescence RT-PCR; 2 . The virus is very homologous to COVID-19 via virus gene sequencing; 3 . Serum COVID-19 specific IgM antibody and IgG antibody were positive; serum COVID-19 specific IgG antibody changed from negative to positive or rising speed in the recovery period was 4 times or more than the acute period. The discharge standard was met the following 4 requirements at the same time: 1 . The body temperature returned to normal for more than 3 days; 2 . Symptoms of respiratory tract greatly improved; 3 . Pulmonary imaging showed that the acute exudation lesions were significantly improved; 4 . The nucleic acid test of respiratory tract samples such as sputum and nasopharyngeal swab was negative for two consecutive time, sampling time shall be at least 24 hours apart. The above 2 criteria referenced "COVID-19 Diagnosis and Treatment Plan (Trial Version 7)" printed and distributed by the National Health Commission of the People's Republic of China on March 4, $2020^{19}$.

\section{Participants evaluation}

Demographic characteristics and psychological health status data were gathered. All participants and researchers were Chinese. Therefore Chinese language was chosen during the whole research process. Demographic data were self-reported by the interviewees, including gender (male or female), age ( $<32$ or 232), classification (COVID-19 survivor, nurse, or hygienist), marriage status (married, or unmarried), and education level (high school and below, college, or bachelor and above).

The SCL90-R questionnaires was composed of 90 items, which were now classified to 9 psychological dimensions listed as: 1. Somatization (SOM); 2. Obsessive-compulsive (O-C); 3. Interpersonal sensitivity (I-S); 4. Depression (DEP); 5. Anxiety (ANX); 6. Anger-hostility (HOS); 7. Phobic anxiety (PHOB); 8. Paranoid ideation (PAR); 9. Psychoticism (PSY). All items were single choice questions, and answers to each entry were rated on a five-point scale from 1 to 5 . Mental health status results corresponding to each score were "none", "mild", "moderate", "relatively serious", and "serious", respectively. The survey was conducted in private environment spending about 25-30 minutes for each interviewee.

\section{Definitions and scores}

Previous studies have demonstrated that the SCL-90-R tests have good internal consistency, reliability, and validity ${ }^{20-23}$. Overall mental health status was evaluated via 3 global scales: 1 . The Global Severity 
Index (GSI), indicating a global scale; 2. Positive Symptom Total (PST), expressing all items more than 1 point to display number of items did the subjects presenting symptoms; and 3. Positive Symptom Distress Index (PSDI), meaning the sum of values of none-zero items by PST, reflecting the extent of severity of items that subjects feel bad about themselves. Higher score indicated more seriously mental health status.

\section{Statistical analysis}

Analysis of all data was performed using the SPSS 25.0 for windows (SPSS Inc., IL, USA). Descriptive analysis was conducted to express the data; continuous variables were expressed as mean \pm standard deviation, while categorical variables were shown as percentages. A chi-squared test was used for statistical analysis and Fisher's exact test when appropriate. $P \leq 0.05$ was considered significant.

\section{Results}

\section{Demographic characteristics}

Demographic items in this study included gender, age, occupation classification, marriage status, educational level. A total of 98 participants were included in the research, who were separated into 3 categories according to occupational classification. Among them, 20 were COVID-19 survivors (20.41\%), 54 nurses $(55.10 \%)$, and 24 hygienists $(24.49 \%$ ) separately. Mean age was $32.1 \pm 7.3$ (range, $23-68$ ), and 68 were female $(69.74 \%)$. Of the marriage status, $24(24.49 \%)$ got married and $74(75.51 \%)$ were unmarried. $66.32 \%(n=65)$ had higher education of the bachelor and above while $20.41 \%(n=20)$ had college education, and the remained $13.27 \%(n=13)$ had a high school education and below (Table 1$)$. 
Table 1

Demographic characteristics of the participants $(n=98)$

\begin{tabular}{|llll|}
\hline Demographic items & Status & Frequency & $\%$ \\
\hline Gender & Male & 30 & 30.61 \\
\cline { 2 - 4 } & Female & 68 & 69.74 \\
\hline Age & $<32$ & 55 & 56.12 \\
\cline { 2 - 4 } & $\geq 32$ & 43 & 43.88 \\
\hline Marriage Status & CoVID-19 Survivor & 20 & 20.41 \\
\cline { 2 - 4 } & Nurse & 54 & 55.10 \\
\cline { 2 - 4 } Education Level & Hygienist & 24 & 24.49 \\
& Unmarried & 24 & 24.49 \\
\cline { 2 - 4 } & High school and below & 13 & 75.51 \\
\cline { 2 - 4 } & College & 20 & 20.41 \\
\cline { 2 - 4 } & University and above & 65 & 66.32 \\
\cline { 2 - 4 } & & & \\
\hline
\end{tabular}

\section{Psychological dimensions}

The overall mean GSI, PST, and PSDI of all subjects was $1.393 \pm 0.391,24.934 \pm 21.463,2.296 \pm 0.620$ respectively. Scores of 9 dimensions were: SOM, 1.366 \pm 0.415 ; O-C, 1.618 $\pm 0.531 ; \mathrm{I}-\mathrm{S}, 1.420 \pm 0.528$; DEP, 1.392 \pm 0.467 ; ANX, 1.401 $\pm 0.432 ;$ HOS, 1.336 $\pm 0.460 ;$ PHOB, $1.233 \pm 0.333$; PAR, $1.268 \pm 0.385 ;$ PSY, $1.303 \pm 0.430$, separately. Among the score, PSDI, ANX, and PSY showed a rising trend (Table 2). 
Table 2

Psychological dimensions of SCL-90-R among the participants $(n=98)$

\begin{tabular}{|c|c|c|c|c|}
\hline \multirow[t]{2}{*}{ Clinical Diagnosis } & \multirow[t]{2}{*}{ Mean Score } & \multirow[t]{2}{*}{ Standard Deviation } & \multicolumn{2}{|l|}{$95 \% \mathrm{Cl}$} \\
\hline & & & Lower & Upper \\
\hline GSI & 1.393 & 0.391 & 1.305 & 1.481 \\
\hline PST & 24.934 & 21.463 & 20.109 & 29.760 \\
\hline PSDI & 2.296 & 0.620 & 2.157 & 2.436 \\
\hline SOM & 1.366 & 0.415 & 1.273 & 1.460 \\
\hline O-C & 1.618 & 0.531 & 1.499 & 1.738 \\
\hline I-S & 1.420 & 0.528 & 1.301 & 1.538 \\
\hline DEP & 1.392 & 0.467 & 1.287 & 1.497 \\
\hline ANX & 1.401 & 0.432 & 1.304 & 1.498 \\
\hline HOS & 1.336 & 0.460 & 1.232 & 1.439 \\
\hline PHOB & 1.233 & 0.333 & 1.158 & 1.308 \\
\hline PAR & 1.268 & 0.385 & 1.181 & 1.354 \\
\hline PSY & 1.303 & 0.430 & 1.206 & 1.399 \\
\hline \multicolumn{5}{|c|}{$\begin{array}{l}\text { Abbreviations: Cl, Confidence interval; GSI, Global Severity Index; PST, Positive Symptom Total; PSDI } \\
\text { Positive Symptom Distress Index; SOM, somatization; O-C, obsessive-compulsive; I-S, interpersonal } \\
\text { sensitivity; DEP, depression; ANX, anxiety; HOS, anger-hostility; PHOB, phobic anxiety; PAR, paranoid } \\
\text { ideation; PSY, psychoticism }\end{array}$} \\
\hline
\end{tabular}

The mean raw score of GSI in COVID-19 survivors, nurses, and hygienists were $1.56 \pm 0.35,1.35 \pm 0.38$, and $1.40 \pm 0.47$. PST were $36.25 \pm 20.25,22.17 \pm 20.93$, and $26.30 \pm 23.24$. PSDI were $2.34 \pm 0.34,2.34 \pm 0.62$, and $1.99 \pm 0.84$. SOM were $1.65 \pm 0.53,1.33 \pm 0.38$, and $1.25 \pm 0.34$. 0 -C were $1.80 \pm 0.47,1.58 \pm 0.53$, and $1.61 \pm 0.59$. I-S were $1.53 \pm 0.49,1.40 \pm 0.54$, and $1.42 \pm 0.55$. DEP were $1.56 \pm 0.38,1.34 \pm 0.42$, and $1.47 \pm 0.75$. ANX were $1.67 \pm 0.45,1.34 \pm 0.41$, and $1.39 \pm 0.43$. HOS were $1.47 \pm 0.41,1.31 \pm 0.47$, and $1.30 \pm 0.47$. PHOB were $1.43 \pm 0.48,1.18 \pm 0.28$, and $1.30 \pm 0.34$. PAR were $1.39 \pm 0.44,1.24 \pm 0.37$, and $1.28 \pm 0.41$. PSY were $1.38 \pm 0.40,1.27 \pm 0.41$, and $1.41 \pm 0.57$, respectively (Table 3 ). In general, the mean raw score of 3 indices and 9 dimensions showed highest in COVID-19 survivors, then the hygienists, and the lowest in nurses. The COVID-19 survivors showed significantly higher scores of PST $(36.25 \pm 20.25$ versus $22.17 \pm 20.93$, $P<0.05)$, SOM ( $1.65 \pm 0.53$ versus $1.33 \pm 0.38, P<0.05), 0-C(1.80 \pm 0.47$ versus $1.58 \pm 0.53, P<0.05)$, ANX (1.67 \pm 0.45 versus $1.34 \pm 0.41, P<0.05)$, and $\mathrm{PHOB}(1.43 \pm 0.48$ versus $1.18 \pm 0.28, P<0.05)$ when compared with nurses. Meanwhile, nurses represented significantly higher in the SOM $(1.33 \pm 0.38$ versus $1.25 \pm 0.34$, $P<0.05)$, and significantly lower in the PHOB $(1.18 \pm 0.28$ versus $1.30 \pm 0.34, P<0.05)$ dimensions when compared with hygienists (Figure 1a, b). 
There were 7 dimensions (SOM, O-C, I-S, ANX, HOS, PAR, and PSY) showing extremely high (mean raw score $\geq 2$ ) in COVID-19 survivors with proportions more than $15 \%$. Moreover, there were two dimensions (O-C and ANX) in hygienists and 1 dimension (O-C) exceedingly high (mean raw score $\geq 2$ ) respectively (Figure 2).

Table 3

Mean raw score on dimensions among the participants $(n=98)$

\begin{tabular}{|llllll}
\hline & COVID-19 Survivors $(n=20)$ & Nurses $(n=54)$ & Hygienists $(n=24)$ & $F$ & $P$ \\
\hline GSI & $1.56 \pm 0.35$ & $1.35 \pm 0.38$ & $1.40 \pm 0.47$ & 1.431 & 0.246 \\
\hline PST & $36.25 \pm 20.25$ & $22.17 \pm 20.93$ & $26.30 \pm 23.24$ & 2.206 & $0.117^{\star}$ \\
\hline PSDI & $2.34 \pm 0.34$ & $2.34 \pm 0.62$ & $1.99 \pm 0.84$ & 1.465 & 0.238 \\
\hline SOM & $1.65 \pm 0.53$ & $1.33 \pm 0.38$ & $1.25 \pm 0.34$ & 3.605 & $0.032^{\star}$ \\
\hline O-C & $1.80 \pm 0.47$ & $1.58 \pm 0.53$ & $1.61 \pm 0.59$ & 0.845 & $0.434^{\star}$ \\
\hline I-S & $1.53 \pm 0.49$ & $1.40 \pm 0.54$ & $1.42 \pm 0.55$ & 0.305 & 0.738 \\
\hline DEP & $1.56 \pm 0.38$ & $1.34 \pm 0.42$ & $1.47 \pm 0.75$ & 1.227 & 0.299 \\
\hline ANX & $1.67 \pm 0.45$ & $1.34 \pm 0.41$ & $1.39 \pm 0.43$ & 2.873 & $0.063^{\star}$ \\
\hline HOS & $1.47 \pm 0.41$ & $1.31 \pm 0.47$ & $1.30 \pm 0.47$ & 0.626 & 0.538 \\
\hline PHOB & $1.43 \pm 0.48$ & $1.18 \pm 0.28$ & $1.30 \pm 0.34$ & 3.206 & $0.046^{*}$ \\
\hline PAR & $1.39 \pm 0.44$ & $1.24 \pm 0.37$ & $1.28 \pm 0.41$ & 0.762 & 0.470 \\
\hline PSY & $1.38 \pm 0.40$ & $1.27 \pm 0.41$ & $1.41 \pm 0.57$ & 0.666 & 0.517 \\
\hline
\end{tabular}

Note: * $p<0.05$.

Abbreviations: Cl, Confidence interval; GSI, Global Severity Index; PST, Positive Symptom Total; PSDI, Positive Symptom Distress Index; SOM, somatization; O-C, obsessive-compulsive; I-S, interpersonal sensitivity; DEP, depression; ANX, anxiety; HOS, anger-hostility; PHOB, phobic anxiety; PAR, paranoid ideation; PSY, psychoticism.

\section{Risk factors of psychological dimensions}

Within the distinction of education level, participants with education of bachelor and above showed significantly lower level of GSI $(1.24 \pm 0.32$ versus $1.37 \pm 0.37, P<0.05)$, PST $(19.00 \pm 25.46$ versus $24.06 \pm 21.19, P<0.05)$, SOM (1.38 \pm 0.53 versus $1.34 \pm 0.40, P<0.05)$, $0-C(1.35 \pm 0.49$ versus $1.59 \pm 0.49$, $P<0.05)$, I-S ( $1.11 \pm 0.16$ versus $1.38 \pm 0.48, P<0.05)$, HOS $(1.17 \pm 0.00$ versus $1.30 \pm 0.42, P<0.05)$, PAR $(1.08 \pm 0.12$ versus $1.23 \pm 0.32, P<0.05)$, and PSY $(1.10 \pm 0.14$ versus $1.28 \pm 0.39, P<0.05)$ compared with college educational level. Meanwhile, participants with college educational level showed significantly 
lower level of PST (19.00 \pm 25.46 versus $49.67 \pm 16.17, P<0.05)$, $0-C(1.35 \pm 0.49$ versus $2.57 \pm 0.85, P<0.05)$, I-S (1.11 \pm 0.16 versus $2.48 \pm 0.76, P<0.05)$, HOS $(1.17 \pm 0.00$ versus $2.28 \pm 0.67, P<0.05)$, PAR $(1.08 \pm 0.12$ versus $2.28 \pm 0.51, P<0.05)$, PSY $(1.10 \pm 0.14$ versus $1.93 \pm 0.95, P<0.05)$ than participants with high school and below education level (Figure $3 a, b)$.

In gender aspects, female subjects expressed significantly higher scores in PSDI $(2.43 \pm 0.56$ versus $2.00 \pm 0.67, P<0.05)$, SOM (1.42 \pm 0.43 versus $1.24 \pm 0.35)$, $0-C$ ( $1.67 \pm 0.58$ versus $1.50 \pm 0.39)$, DEP (1.43 \pm 0.53 versus $1.31 \pm 0.29)$, and HOS $(1.37 \pm 0.49$ versus $1.26 \pm 0.39)$ compared with male participants (Figure 4a, b).

No statistical significance was discovered among participants within different age (Figure 5a, b) or marriage status (Figure 6a, b) for each aspect and dimension of the measurements.

\section{Discussion}

The COVID-19 outbreak was thought to be the most prevalent and serious epidemic since the end of December of 2019. Over 200 countries, areas or territories were suffering from the virus and more than 2 million persons infected by the acute infectious disease with over 130 thousand confirmed death globally $6^{6}$. Owing to the significant morbidity and mortality, the COVID-19 caused large scale public panic and serious psychological health stress ${ }^{24}$. The WHO had emphasized the importance of psychological support and released some advice to survivors and health care workers ${ }^{25-27}$. However, to our knowledge, there is only a few researches focusing on mental health of COVID-19 survivors and frontline healthcare workers. Thus it is necessary to conduct scientific research to evaluate the mental health status of the COVID-19 outbreak. This study was to assess psychological health status of COVID-19 survivors, nurses, and hygienists in Wuhan, China, analyze the risk factors to influence the outcome, and provide help for mental health assistance for survivors and healthcare workers.

Results of the present research indicated that psychological health status among all participants in Wuhan, China was not very serious, and the main problems were anxiety and psychoticism. Possible reasons may be as follows: 1 . Our assessment time was set on over 2 months after the outbreak when the worst situation has passed and under controlled effectively in Wuhan, China. The COVID-19 has been intensively studied through advanced medical scientific techniques and released to the public in time, making participants very familiar with the new virus and reducing their fear of the new virus; 2 . The Chinese government and some medical organizations have taken a serious of measures for psychological support to the confirmed patients and healthcare professionals ${ }^{28,29} ; 3$. Latest daily report was released by authorities, thus the public could receive the specific trends of the epidemic situation. In addition, energy propaganda was carried out by the mainstream media to fight the virus epidemic, encouraging everyone to face it positively; 4 . The epidemic crisis was under control in China, and there was still newly increased confirmed death cases. What's more, the epidemic was further expanded in other countries ${ }^{5}$. Under the background of globalization, and a community of sharing future for mankind, participants still felt anxiety to the COVID-19 and showed poor mental status. 
Our studies showed that COVID-19 survivors suffered severe somatization, obsession-compulsion, interpersonal sensitivity, anxiety, anger-hostility, paranoid ideation, psychoticism, and highest PST score which meant they had worse psychological status compared with healthcare workers. This was consistent with a previous study about comparison of psychological symptoms between Ebola survivors and healthcare workers ${ }^{30}$. Possible reasons were maybe the novel virus was characterized by a high possibility of being infected, and high mortality which was a life threatening event ${ }^{31}$. Patients were forced isolation in specific hospitals, which divided them from work, family, and conventional daily life, so that to plumb the depths of loneliness, humiliation, and even despair ${ }^{32}$. Besides, some bad media reported false news to mislead public which aggravated patients' panic and anxiety ${ }^{33}$. Results analysis showed that it was important to construct an appropriate emergency response mechanism of serious infectious disease and recovery system of post-traumatic stress. Specific methods included establishing professional psychological intervention team which consisted of all mental health professionals, conducting online psychological counselling services (eg, on WeChat), etc $7,8,10$. Under the guidance of government ${ }^{34}$, we were required to pay attention to patients' mental health and psychological response. Psychological evaluation, counseling, and supporting should be provided to recovered patients.

This study showed that psychological status of healthcare workers including nurses and hygienists was not very serious generally, and nurses presented higher score in somatization than hygienists. It was comparable to the previous study about comparison of hygienists and nurses during the COVID-19 crisis $^{35}$. Possible reasons probably were nurses contacted with patients directly and frequently with wearing sealed personal protective equipment which made them sultry, dyspnea, facial skin indentation or even damaged ${ }^{36}$. What was worse, they need to work longer hours than usual without break or going to the toilet, causing high risk of infection. Physical fatigue made a damage to the body and performed severe physical symptoms ${ }^{37}$. However, nurses presented lower score in phobic anxiety than hygienists which was contrary to previous study ${ }^{35}$. To our consideration, reason for this result may be the hygienists were responsible for making treating plan for patients, and they need to be very careful in each step because of the high mortality. Facing too many death and feeling powerless may lead to phobic anxiety. Though healthcare workers' mental health was not serious, attention should be paid to increasing levels of somatization, obsession-compulsion, anxiety, and phobic anxiety. Psychological counselling, disaster rescue knowledge training and simulation exercise, formulation of relevant processes and emergency plans should be implemented ${ }^{13}$.

In this study, there was a negative correlation between the severity of mental state and education level, which is consistent with a previous study ${ }^{30}$. These findings indicated education was an import and effective aspect leading people to know virus correctly and confront it positively. People with higher education level might think more about the accepted news and information, instead of believing every information they received, even rumors and false beliefs. More proper education of the novel virus applied, less mental problems they would have. Thus, we need to construct proper educational channels of major infectious disease to the public. 
Of note, female participants showed worse PSDI, somatization, obsessive-compulsive, depression, and anger-hostility. It was consisted with a previous study that women had more mental problems in the COVID-19 outbreak ${ }^{35}$. Reasons may be in face of sudden changes, women can't be as calm as men. Women usually had rich inner activities as facing such an emergency crisis, and they worried about their family members or daily social interaction. This indicated us to take particular attention to female survivors and healthcare workers, more family and social support, and psychological intervention was warranted.

We also interested in whether there was divergence in different ages and marriage status. The results indicated no significant difference possibly because: Within this sudden highly infectious virus, everybody had the possibility of infection and death. The junior or senior, married or unmarried, were similar in the face of the COVID-19.

\section{Conclusions}

In this survey study of COVID-19 survivors and healthcare workers in Wuhan, China, mental distress is not very serious in general. Yet, specific methods have to be implemented to promote survivors' mental well-being immediately. Meanwhile, healthcare workers warrant more attention and intervention particularly. Furthermore, low-educated, and women require meticulous attention. To solve these problems, we call for a comprehensive emergency response plan and to be developed involving not only medical facilities but also adequate psychological support.

\section{Limitations}

This study presents several limitations. Firstly, the sample size is small. Because the crisis outbreak was still ongoing during the research period, we should allow medical staff to spend more time on patients or rest. In addition, the study was carried out during 10 days in the late stage of fighting epidemic in Wuhan, China, while the early, middle stage and longitudinal follow-up were not considered. Thus we couldn't investigate the dynamic psychological change. Further studies with larger sample size and observation of multiple time point are warranted.

\section{References}

1.Li Q, Guan X, Wu P, et al. Early transmission dynamics in Wuhan, China, of novel coronavirus-infected pneumonia. [Epub ahead of print]. N Engl J Med. 2020.

2.Lu HZ, Stratton CW, Tang YW. Outbreak of pneumonia of unknown etiology in Wuhan, China: the mystery and the miracle. J Med Virol. 2020;92(4):401-402.

3.Khan S, Ali A, Siddique R, Nabi G. Novel coronavirus is putting the whole world on alert. J Hosp Infect. 2020;104(3):252-253. 
4.Kang L, Li Y, Hu S, et al. The mental health of medical workers in Wuhan, China dealing with the 2019 novel coronavirus. The Lancet Psychiatry. 2020;7(3):e14.

5.National Health Commission of the People's Republic of China. Updates on the novel coronavirus outbreak up to April 22, 2020. Accessed April 23, 2020.

http://www.nhc.gov.cn/xcs/yqfkdt/202004/ab35f22c371149ad95284b1539f2a8a6.shtml.

6.World Health Organization. Coronavirus disease 2019 (COVID-19) Situation Report-93.

https://www.who.int/docs/default-source/coronaviruse/situation-reports/20200422-sitrep-93-covid19.pdf?sfvrsn $=35 \mathrm{cf} 80 \mathrm{~d} 7 \_4$.

7.Bao Y, Sun Y, Meng S, Shi J, Lu L. 2019-nCoV epidemic: address mental health care to empower society. The Lancet. 2020;395(10224):e37-e38.

8.Xiao C. A novel approach of consultation on 2019 novel coronavirus (COVID-19)-related psychological and mental problems: structured letter therapy. Psychiatry Investig. 2020;17(2):175-176.

9.Bo HX, Li W, Yang Y, et al. Posttraumatic stress symptoms and attitude toward crisis mental health services among clinically stable patients with COVID-19 in China. Psychol Med. 2020:1-7.

10.Liu S, Yang L, Zhang C, et al. Online mental health services in China during the COVID-19 outbreak. The lancet Psychiatry. 2020;7(4):e17-e18.

11.Mak IWC, Chu CM, Pan PC, Yiu MGC, Chan VL. Long-term psychiatric morbidities among SARS survivors. Gen Hosp Psychiatry. 2009;31(4):318-326.

12.Chen Q, Liang M, Li Y, et al. Mental health care for medical staff in China during the COVID-19 outbreak. The lancet Psychiatry. 2020;7(4):e15-e16.

13.Greenberg N, Docherty M, Gnanapragasam S, Wessely S. Managing mental health challenges faced by healthcare workers during covid-19 pandemic. BMJ. 2020;368:m1211.

14.National Health Commission of China. A notice on the issuance of guidelines for emergency psychological crisis intervention in pneumonia for novel coronavirus infections.

http://www.nhc.gov.cn/xcs/zhengcwj/202001/6adc08b966594253b2b791be5c3b9467.shtml.

15.Chen QN, Liang MN, Li YM, et al. Mental health care for medical staff in China during the COVID-19 outbreak. The Lancet Psychiatry. 2020;7(4):e15-e16.

16.Duan L, Zhu G. Psychological interventions for people affected by the COVID-19 epidemic. The Lancet Psychiatry. 2020;7(4):300-302.

17.Dudley J, Richards L, Mahmud M. The use of a psychological testing instrument as an indicator of dissatisfaction with aesthetic dental treatment-a preliminary study. BMC psychology. 2020;8(1):24. 
18.Citkowska-Kisielewska A, Rutkowski K, Mielimąka M, Sobański J, Dembińska E. Obsessive-Compulsive Symptoms in Obsessive-Compulsive Disorder and in Generalized Anxiety Disorder: Occurrence and Correlations. J Psychiatr Pract. 2020;26(2):101-119.

19.National Health Commission of the People's Republic of China. Notice on COVID-19 diagnosis and treatment plan (trial version 7).

http://www.nhc.gov.cn/xcs/yqtb/202003/c588ee20113b4136b27f2a07faa7075b.shtml.

20.Ringqvist A, Bech P, Glenthoj B, Petersen E. Acute and long-term psychiatric side effects of mefloquine: A follow-up on Danish adverse event reports. Travel Med Infect Dis. 2015;13(1):80-88.

21.Palmieri VO, Santovito D, Margari F, Lozupone M, Palasciano G. Psychopathological profile and healthrelated quality of life (HRQOL) in patients with hepatocellular carcinoma (HCC) and cirrhosis. Clinical Experimental Medicine and Surgery. 2013;15(1):65-72.

22.Loesch DZ, Bui MQ, Hammersley E, et al. Psychological status in female carriers of premutation FMR1 allele showing a complex relationship with the size of CGG expansion. Clin Genet. 2015;87(2):173-178.

23.Derogatis LR. Symptom Checklist-90-Revised. In: American Psychiatric Association, ed. Handbook of psychiatric measures. Washington, DC: American Psychiatric Association. 2000: 81-84.

24.Kavoor AR. COVID-19 in people with mental illness: challenges and vulnerabilities. Asian J Psychiatr. 2020;51:102051.

25.Cui L, Wang X, Wang H. Challenges facing coronavirus disease 2019: psychiatric services for patients with mental disorders. [Epub ahead of print]. Psychiatry clinical neurosciences. 2020.

26.Mo Y, Deng L, Zhang L, et al. Work stress among Chinese nurses to support Wuhan for fighting against the COVID-19 epidemic. [Epub ahead of print]. J Nurs Manag. 2020.

27.Bansal P, Bingemann T, Greenhawt M, et al. Clinician wellness during the COVID-19 pandemic: extraordinary times and unusual challenges for the allergist/immunologist [Epub ahead of print]. The journal of allergy clinical immunology in practice. 2020.

28.National Health Commission of the People's Republic of China. Psychological counseling for novel coronavirus pneumonia.

http://www.nhc.gov.cn/jkj/s3578/202003/6348d1d3621e44ccae0d9d40cd5f527a.shtml.

29.Kang L, Li Y, Hu S, et al. The mental health of medical workers in Wuhan, China dealing with the 2019 novel coronavirus. The lancet Psychiatry. 2020;7(3):e14.

30.Ji D, Ji Y, Duan X, et al. Prevalence of psychological symptoms among Ebola survivors and healthcare workers during the 2014-2015 Ebola outbreak in Sierra Leone: a cross-sectional study. Onco Targets Ther. 2017;8(8):12784-12791. 
31.Webster P. Virtual health care in the era of COVID-19. Lancet Infect Dis. 2020;395(10231):1180-1181.

32.Yao H, Chen JH, Xu YF. Patients with mental health disorders in the COVID-19 epidemic. The Lancet Psychiatry. 2020;7(4):e21.

33.Chen Q, Min C, Zhang W, Wang G, Ma X, Evans R. Unpacking the black box: How to promote citizen engagement through government social media during the COVID-19 crisis. [Epub ahead of print]. Comput Human Behav. 2020:106380.

34.National Health Commission of the People's Republic of China. Novel coronavirus pneumonia, isolation and family members psychological counseling and social work service program interpretation materials. http://www.nhc.gov.cn/jkj/s5888/202004/32701ef2e5c940cb8e0e416dbabb4a4d.shtml. 2020.

35.Lai J, Ma S, Wang Y, et al. Factors associated with mental health outcomes among health care workers exposed to coronavirus disease 2019. JAMA Network Open. 2020;3(3):e203976.

36.Gheisari M, Araghi F, Moravvej H, Tabary M, Dadkhahfar S. Skin reactions to non-glove personal protective equipment: an emerging issue in the COVID-19 pandemic. [Epub ahead of print]. Journal of the European Academy of Dermatology Venereology 2020.

37. Li L,Cheng S,Gu J. SARS infection among healthcare workers in Beijing, China. JAMA. 2003; 290(20): 2662-2663. 2020.

\section{Figures}

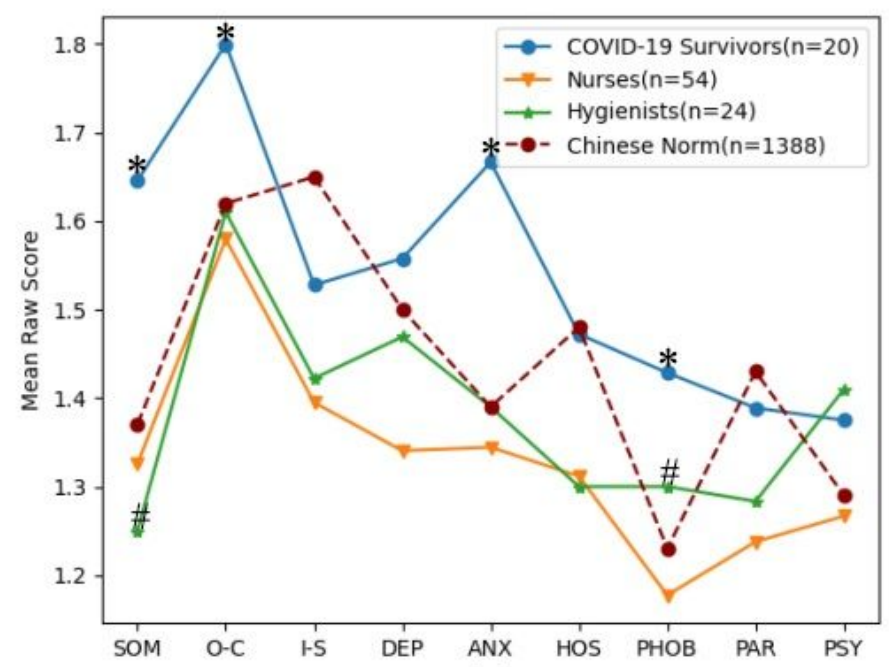

(a)

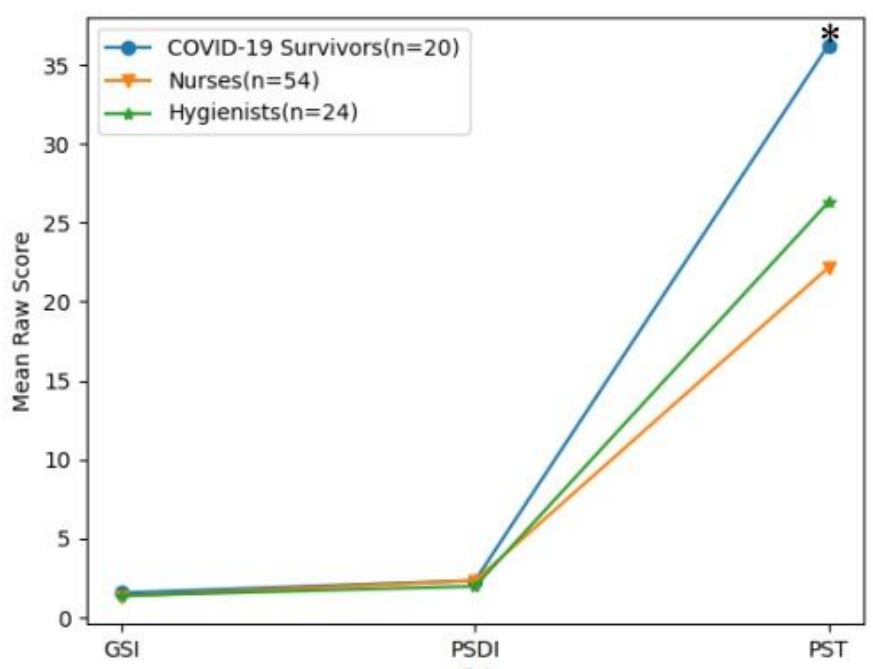

(b)

${ }^{*} \mathrm{p}<0.05$ COVID-19 Survivors vs Nurses $\# \mathrm{p}<0.05$ Nurses vs Hygienists 
Figure 1

Scores of distributions of participants

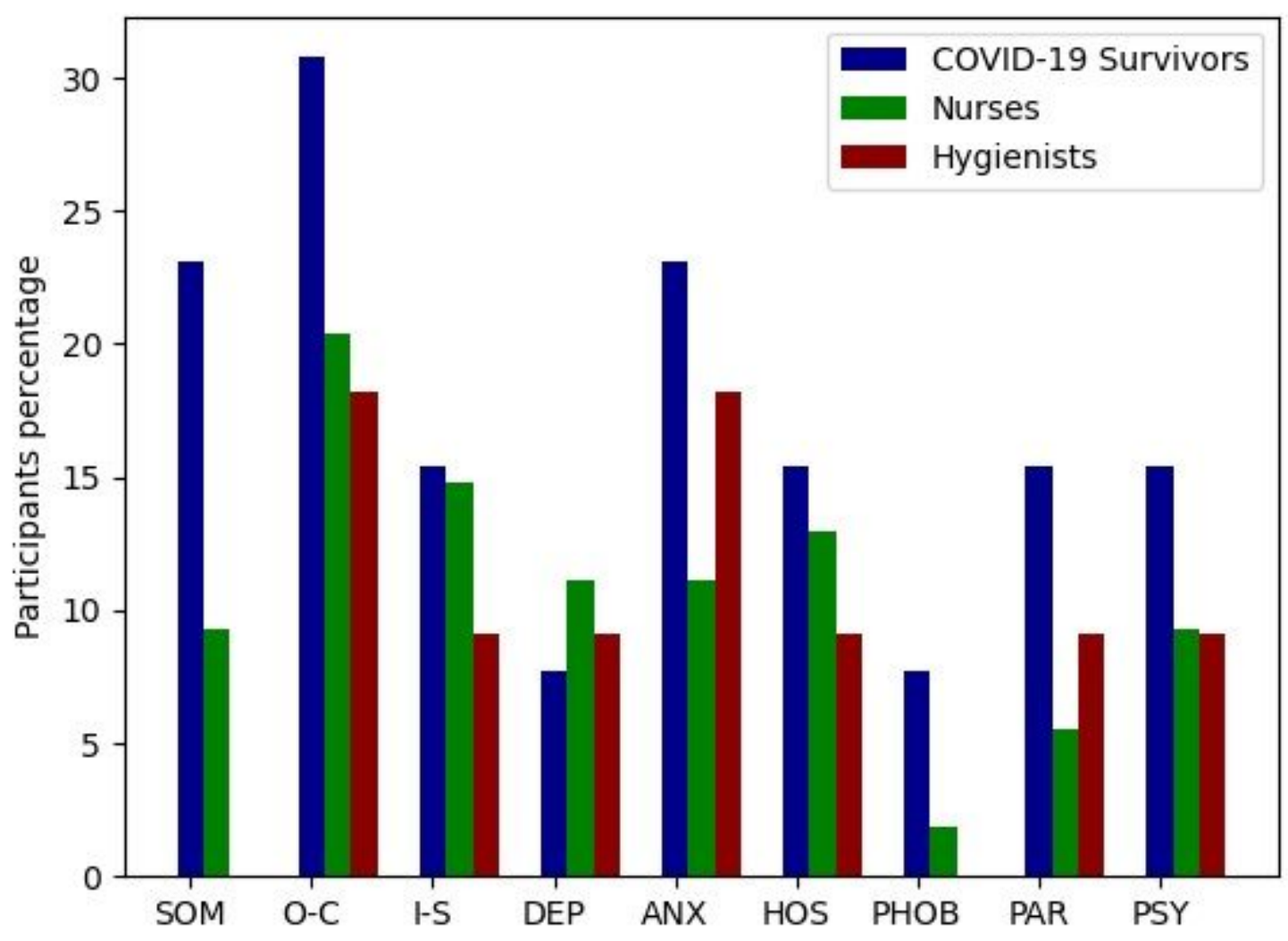

Figure 2

renamed_29663 


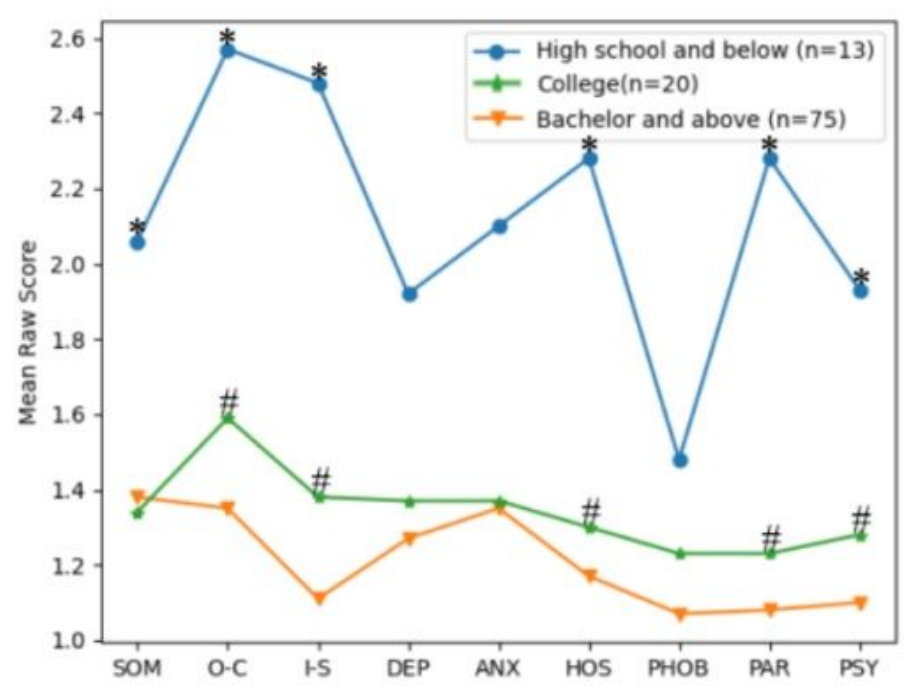

(a)

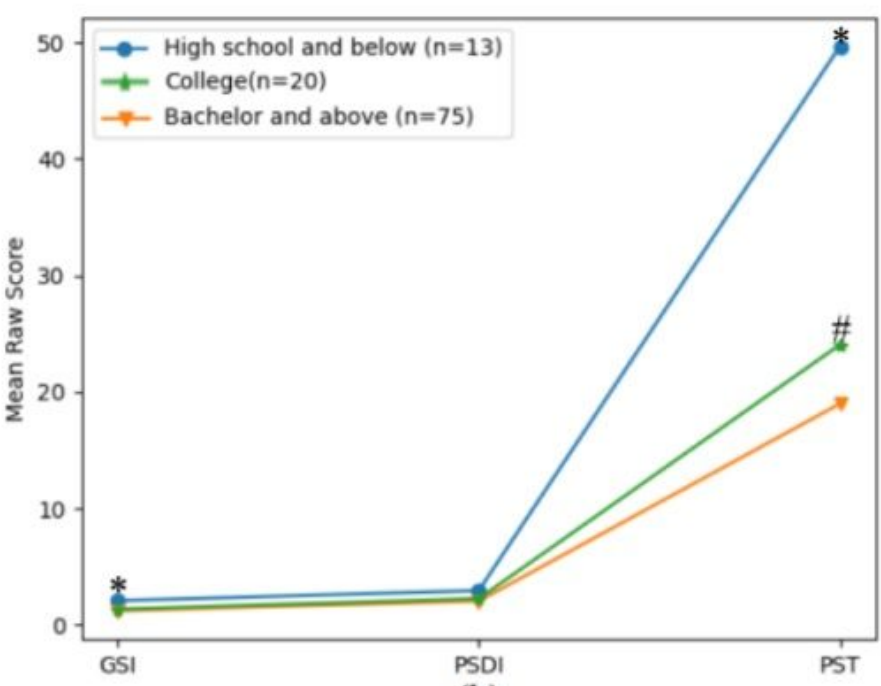

(b)

${ }^{*} \mathrm{p}<0.05$ High school and below vs College

$\# \mathrm{p}<0.05$ College vs Bachelor and above

\section{Figure 3}

Scores of education difference

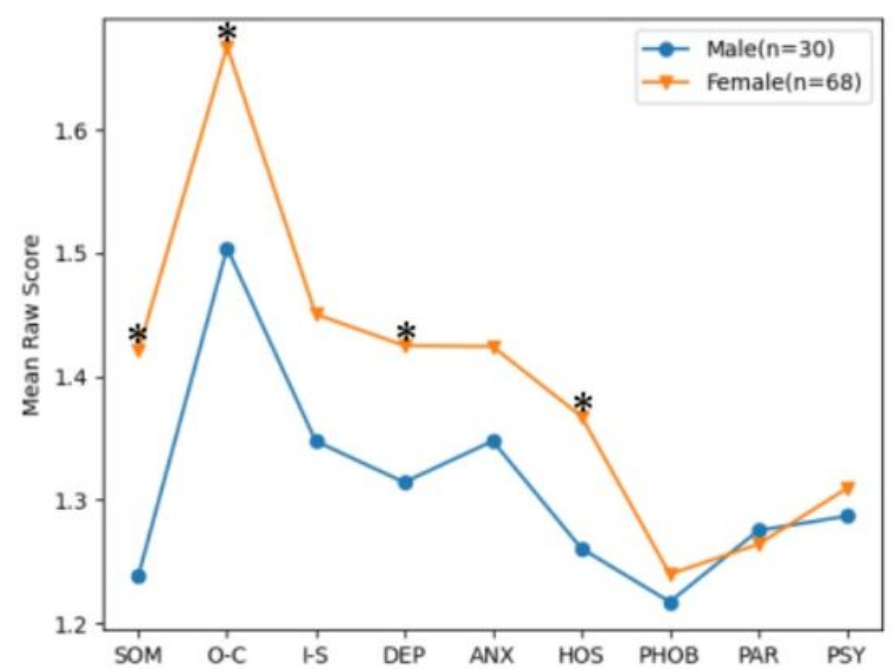

(a)

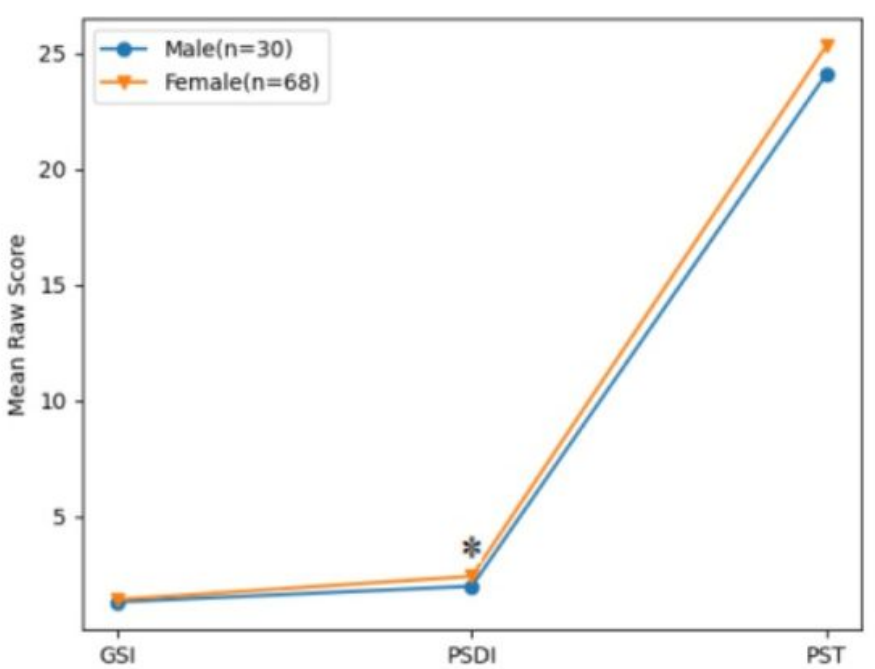

(b)

*p $<0.05$ Male vs Female

Figure 4

Scores of gender difference 


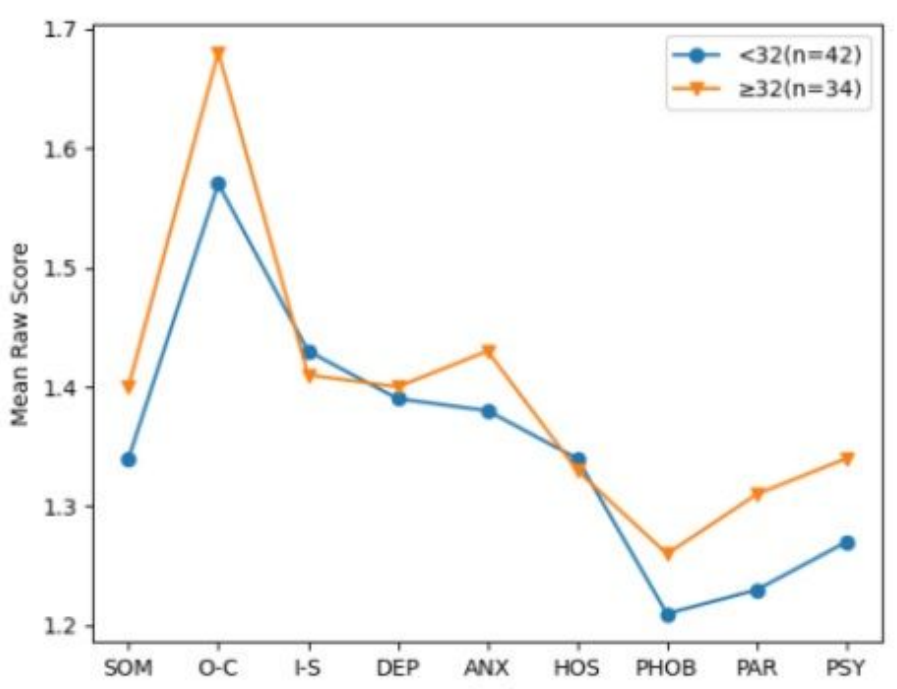

(a)

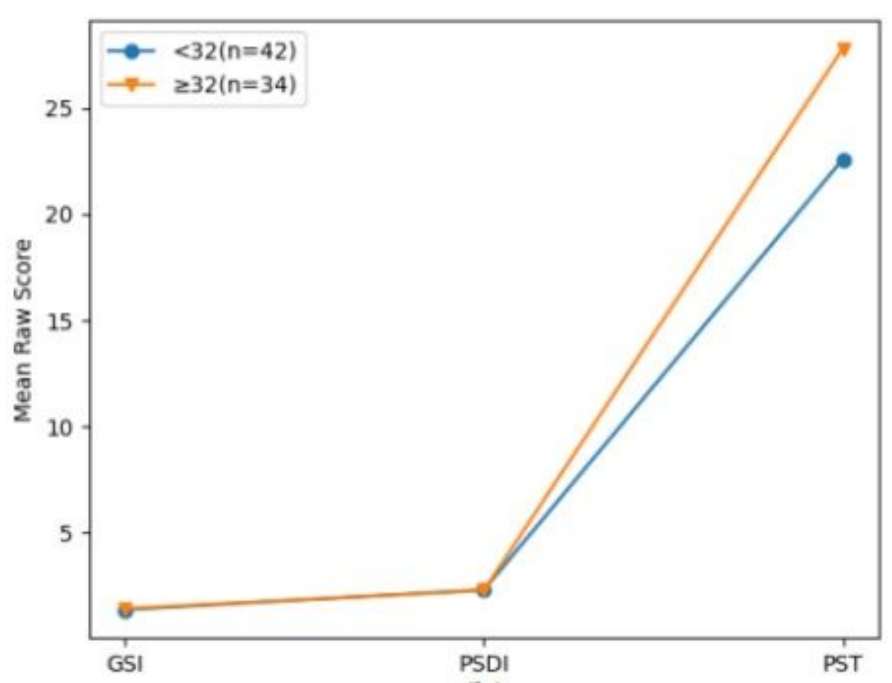

(b)

\section{${ }^{*} \mathrm{p}<0.05$ Age $<32$ vs Age $\geq 32$}

\section{Figure 5}

Scores of age difference

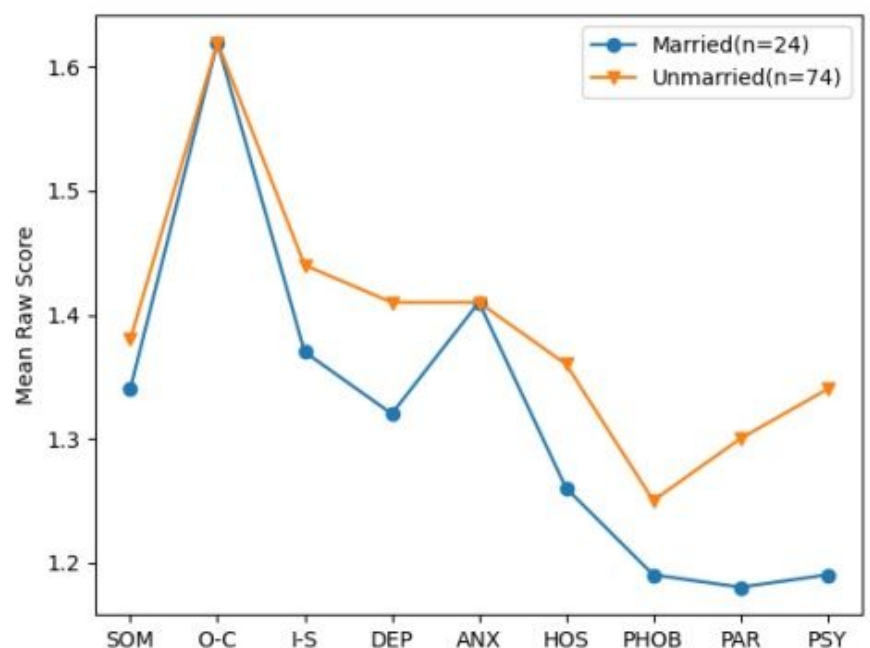

(a)

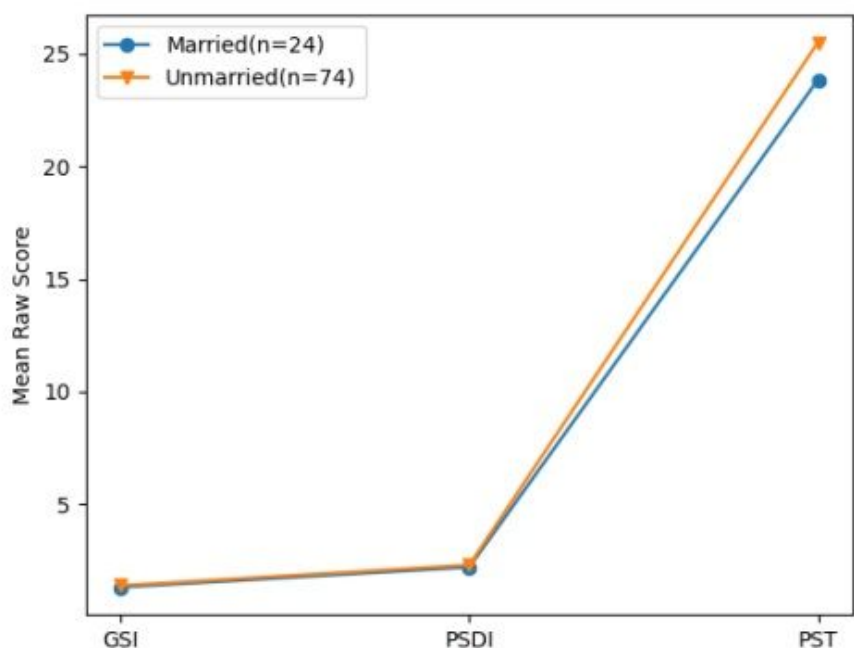

(b)

* $\mathrm{p}<0.05$ Married vs Unmarried

Figure 6

Scores of marriage difference 